\title{
Numerical Study of Heat Transfer and Contaminant Transport in an Unsaturated Porous Soil
}

\author{
Abdelhamid Belghit ${ }^{*}$, Mustapha Benyaich ${ }^{2}$ \\ ${ }^{1}$ Laboratory of Engineering Science for Environment-LaSIE FRE-CNRS 3474, University of La Rochelle, \\ La Rochelle, France \\ ${ }^{2}$ Department of Physics, Faculty of Science, University Cadi Ayyad, Marrakesh, Morocco \\ Email: abelghit@univ-Ir.fr
}

Received 28 July 2014; revised 25 August 2014; accepted 16 September 2014

Copyright (C) 2014 by authors and Scientific Research Publishing Inc.

This work is licensed under the Creative Commons Attribution International License (CC BY). http://creativecommons.org/licenses/by/4.0/

(c) (i) 0pen Access

\section{Abstract}

Penetration of chemicals in the soil ground through irrigation water or rainfall induces important risks for the environment. These risks are badly known and may lead to direct contamination of the environment (atmosphere or ground water) or harmful effects on organisms living at ground level, indirectly affecting men. It is thus necessary to estimate these potential chemical risks on the environment. For that reason, the gradual change of these products (fertilizers, solutions, pollutants, ...) in the ground has been the subject of a lot of recent research works, based in particular on the study of non-saturated porous media in a theoretical, numerical or experimental way. Most of these works are incomplete and, in order to simplify the problem, they don't take into accounts some process, which may be of prime importance under particular natural conditions. Complexity of such studies results from their multidisciplinary nature. In this communication, we study simultaneous transport of pollutant, the water that provides transport and the heat transfer in a $200 \mathrm{~cm}$ long cylindrical column full of sand taken as a non-saturated porous medium. We consider two kinds of conditions on the temperature at the column surface: the case of constant temperature and the case of sinusoidal temperature. We evaluate the influence of this temperature on the transfers. This study is purely numerical. We use the control volume method to determine hydrous, thermal and pollutant concentration profiles.

\section{Keywords}

Soil, Heat and Mass Transfer, Porous Media, Contaminant

\footnotetext{
${ }^{*}$ Corresponding author.
} 


\section{Introduction}

The transfers in solutions in the ground result from a set of phenomena whose knowledge should make it possible to improve management of the irrigation and spreading in agricultural medium, double point of view of the prevention of the contamination of underground water and economy of fertilisers.

Among these phenomena, the purely physical aspect of the transfers constitutes a whole of convection and dispersion mechanisms whose study, in natural environment, is essential for the comprehension of pollutant evolution in the ground.

During these last decades, the study of the vertical and horizontal isothermal transfers of a contaminant in an unsaturated homogeneous porous medium has been the object of an important literature treating the numerical [1]-[6] and experimental [4] [7]-[10] aspect.

However, the majority of these authors considered that the temperature on the surface of the ground is constant [4]. In fact, the temperature of the ground presents continual variations under the influence of the climatic conditions, which are carried out in a relatively stable way according to periodic cycles. These cycles, daily and annual, are indeed connected with the variations of intensity of the solar radiation which appear over some daily periods, by the alternation of the day and the night, and over annual periods, by the evolution of the average slope of the sun on the horizon. The sinusoidal approximation constitutes the simplest manner to describe mathematically a periodic behavior.

So we are going to solve numerically the coupled and non-linear equations governing these transfers, by the method of control volume worked out by Patankar [11].

The results will be given in the form of curves representing the evolution of the hydrous, thermal and concentration profiles. The object of these results is to contribute to the prediction and the control of the pollution of the ground water.

In this work, we will use two different boundary conditions of temperature. The first one is constant and equal to $40^{\circ} \mathrm{C}$; the other one is sinusoidal given by real climatic measurements of Marrakesh (Morocco).

\section{Mathematical Model}

The equations of transfer of water, heat and concentration are given respectively by the model of Philip and Devries [12] and the equation of convection-dispersion, which under certain conditions [13], can be written under the following form:

$$
\begin{array}{r}
\frac{\partial \theta}{\partial t}=\frac{\partial}{\partial z}\left[D_{\theta} \frac{\partial \theta}{\partial z}+D_{T} \frac{\partial T}{\partial z}-K(\theta)\right] \\
\rho C_{p} \frac{\partial T}{\partial t}=\frac{\partial}{\partial z}\left[\lambda \frac{\partial T}{\partial z}\right] \\
\theta \frac{\partial C}{\partial t}=\frac{\partial}{\partial z}\left[D_{a p} \theta \frac{\partial C}{\partial z}\right]-q_{\theta} \frac{\partial C}{\partial z}
\end{array}
$$

The thermohydric coefficients used were evaluated using the experimental data collected by Crausse [14].

\section{Initial and Boundary Conditions}

To solve these equations it is necessary to know the initial and boundary conditions, we propose the following Dirichlet type conditions (Figure 1).

a) For constant surface temperature

$$
\begin{aligned}
& \text { At } t=0\left\{\begin{array}{l}
T=T_{0} \\
\theta=\theta_{0} \\
C=0
\end{array}\right. \\
& \text { at } Z=0 \text { and } t>0\left\{\begin{array}{l}
T=T_{1} \\
\theta=\theta_{s} \\
C=C_{0}
\end{array}\right.
\end{aligned}
$$




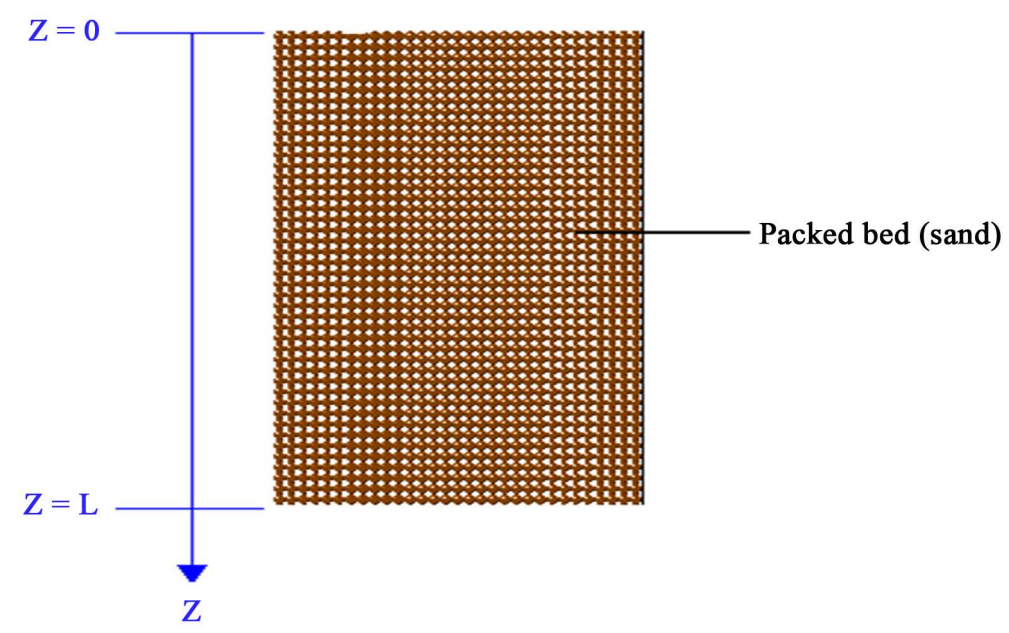

Figure 1. The column scheme.

$$
\text { at } Z=L \text { and } t>0\left\{\begin{array}{l}
-\lambda \frac{\partial T}{\partial z}=0 \\
-D_{\theta} \frac{\partial \theta}{\partial z}-D_{T} \frac{\partial T}{\partial z}+K=0 \\
\frac{\partial C}{\partial z}=0
\end{array}\right.
$$

b) For variable surface temperature

For the resolution of the equation of heat, the values of the temperature on the surface are given by Ouardi et al. [15]:

$$
T(z, t)=T_{0}+\theta_{0} \mathrm{e}^{-k z} \cos (\omega t-k z)
$$

Who used the annual climatic data of Marrakech of the time temperature, to choose 10 days known as standard days, as seen in Figure 2.

c) Equation of moisture

The column is subjected to a constant flow in take making it possible to carry it to a saturation moisture. The other end is maintained at initial moisture:

$$
\begin{gathered}
\text { At time }: t=0 \quad \theta=\theta_{0} \\
\text { At } Z=0 \quad \theta=\theta_{s} \\
\text { At } Z=L \quad \theta=\theta_{0}
\end{gathered}
$$

The contribution of aqueous solutions can be continuous, the case of a table cloth polluted on the surface of the ground, as it can be in crenel, precipitation or irrigation with use of a fertilizer, by considering the time of injection of flow of infinite or finished aqueous solutions $t_{m}$ :

$$
\begin{gathered}
0<t<t_{m} \quad C(X, z, t)=C_{0} \\
t>t_{m} \quad C(X, z, t)=0
\end{gathered}
$$

d) Equation of the concentration

For the equation of concentration we suppose that the surface is maintained at a concentration in solution $C_{0}$, whereas the field is initially deprived of any aqueous solution:

$$
\begin{gathered}
\text { At time : } t=0 \quad C=0 \\
\text { At } Z=0 \quad C=C_{0} \\
\text { At } Z=L \quad C=0
\end{gathered}
$$




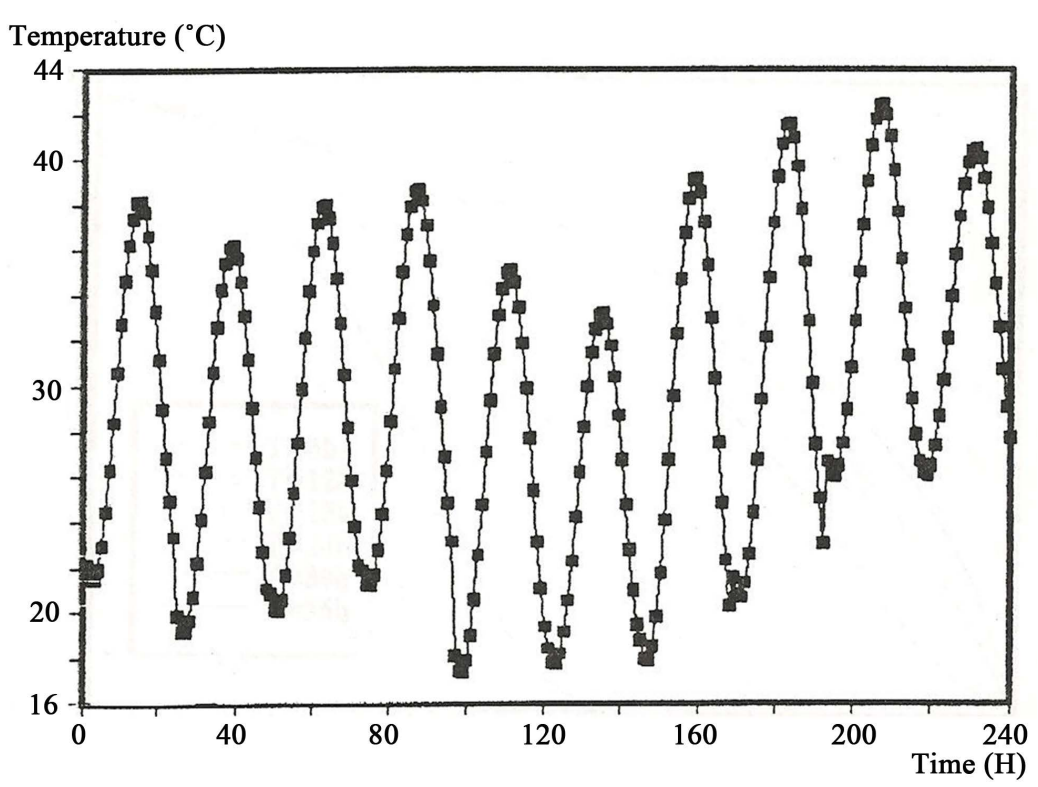

(a)

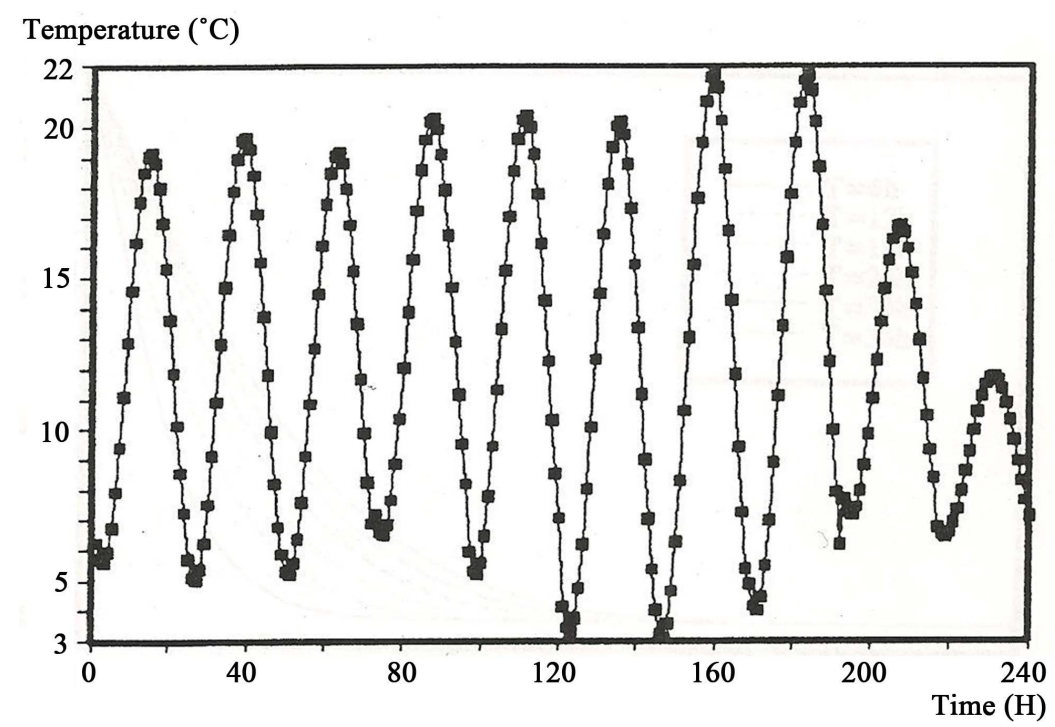

(b)

Figure 2. Values of the surface temperature taken from the annual climatic data of Marrakesh (Morocco) of the time given by Ouardi et al. [15], (a) Summer; (b) Winter.

\section{Numerical Simulation}

The numerical method used is that of the control volume proposed by Patankar [11]. It consists of the discretisation of the field by juxtaposition of elementary volumes inside which we integrates the equations to have them directly discretised. The temperature is periodic of period 24 hours. To show its influence on the transfers it is necessary to make simulations at least equal to this period. In this work, the time of simulation is 36 hours.

The parameters used are:

$\Delta z=0.5 \mathrm{~cm}$ step of space; $\Delta t=6 \mathrm{~s}$, step of time; $\theta_{0}=0.06 \mathrm{~cm}^{3} / \mathrm{cm}^{3} ; \theta_{s}=0.35 \mathrm{~cm}^{3} / \mathrm{cm}^{3} ; T_{0}=293 \mathrm{~K} ; T_{1}=313$ $\mathrm{K} ; C_{0}=10 \mathrm{mg} / \mathrm{l}$ concentration of solute at the entry of column; $D_{a p}=\alpha\left(q_{\theta} / \theta\right)+a \cdot \mathrm{e}^{b \theta}$ and $\alpha=0.2 \cdot L \cdot\left(1-\mathrm{e}^{(-0.05 z / L)}\right)$, a and bare constants that depend on the nature of the soil [16]. 


\section{Results}

In Figure 3 is presented the humidity variations with time and depth for a constant surface temperature. It takes $50 \mathrm{~min}$ to change the humidity at $40 \mathrm{~cm}$ of depth. The velocity of humidity penetration decreases with time due to the soil saturation.

Figure 4 shows the temperature evolution with time and depth, the profiles are similar to the humidity, but the velocity is much lower, this difference is principally to the small soil thermal conduction compared to the humidity dispersion.

The concentration of the inert pollutant is shown in Figure 5. For layers nearer to the soil surface the pollutant diffusion is fast, but for bigger depth the concentration changes are slower, and it changes its concentration only at $30 \mathrm{~cm}$ after $50 \mathrm{~min}$.

Figure 6 shows the variation of temperature at various depths for one day in summer and winter. It is noticed that all the curves have a sinusoidal form but they present a dephasing compared to that of soil surface. With the increase in depth, the layers are less influenced by the heat transfer, thus their time to increase/decrease the temperature is bigger than in the soil surface. These results are in good agreement with those found by [3].

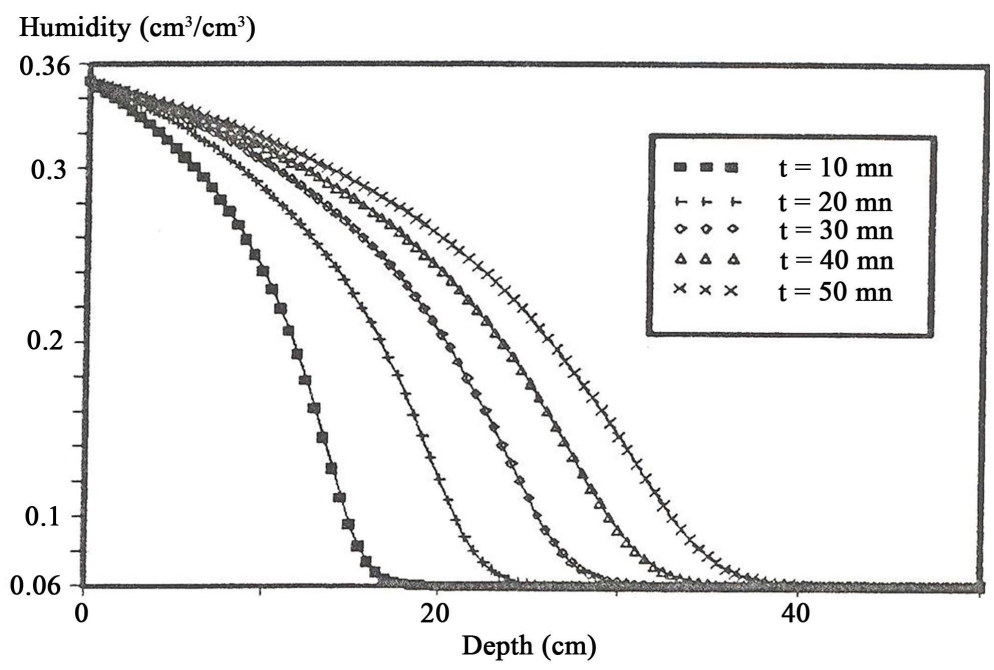

Figure 3. Humidity profiles with depth and time for a constant surface temperature.

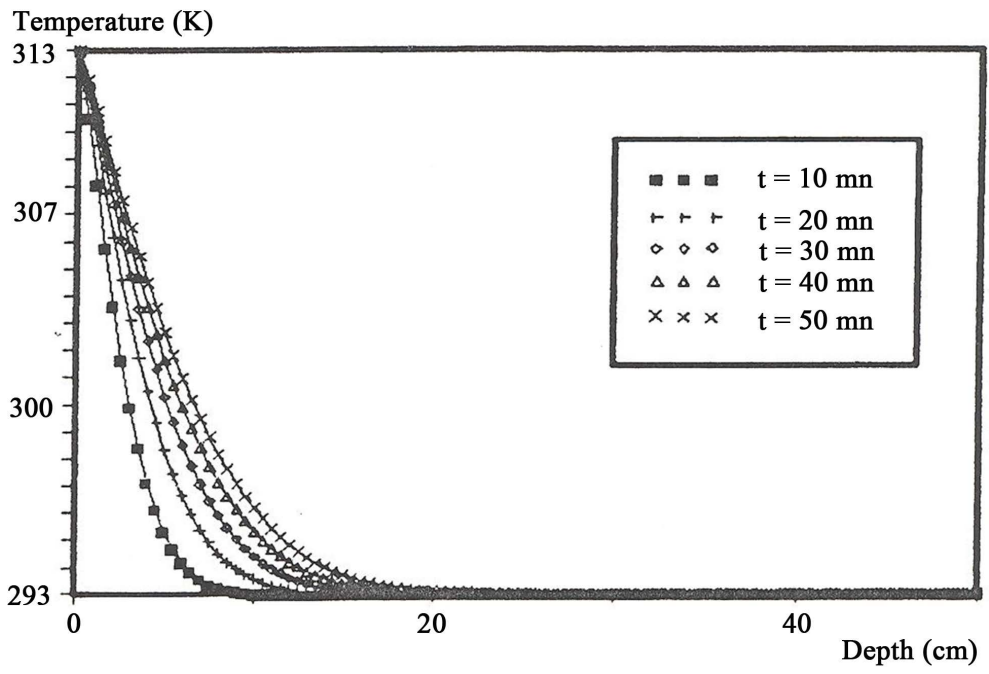

Figure 4. Temperature profiles with depth and time for a constant surface temperature. 


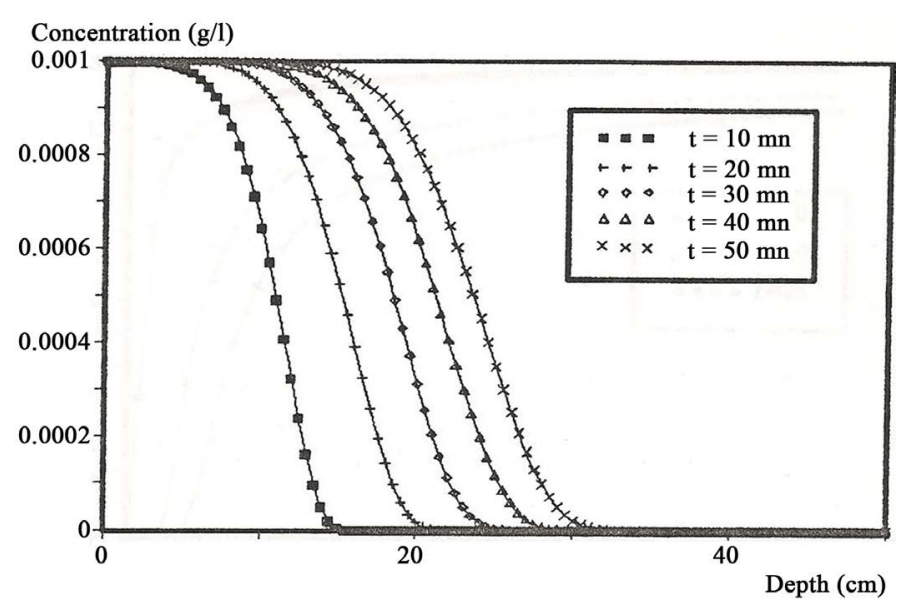

Figure 5. Pollutant concentration profiles with depth and time for a constant surface temperature.

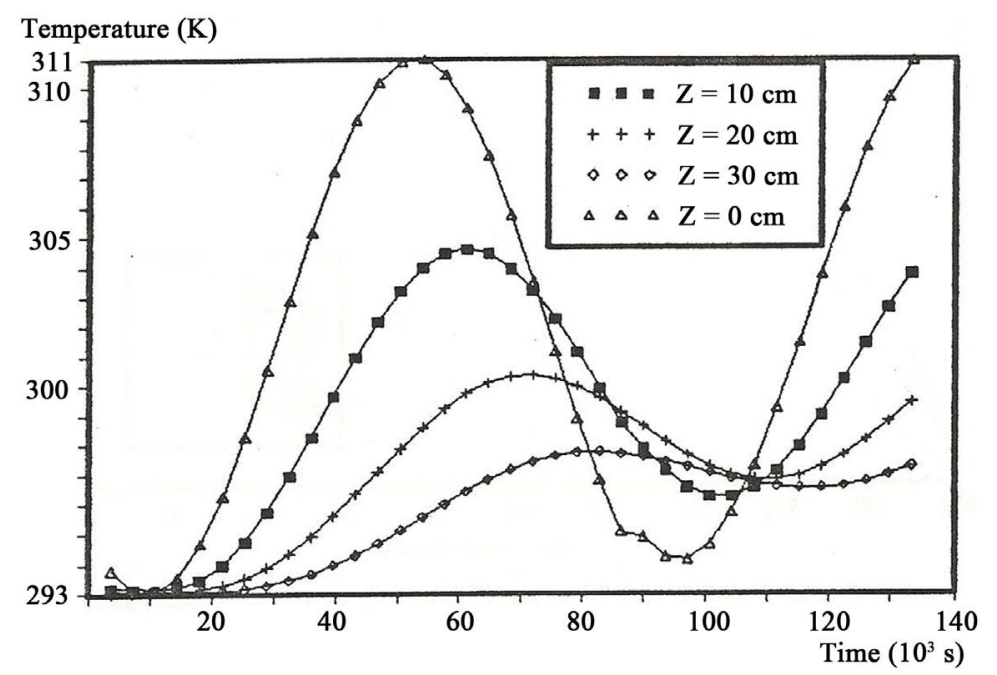

(a)

Temperature (K)

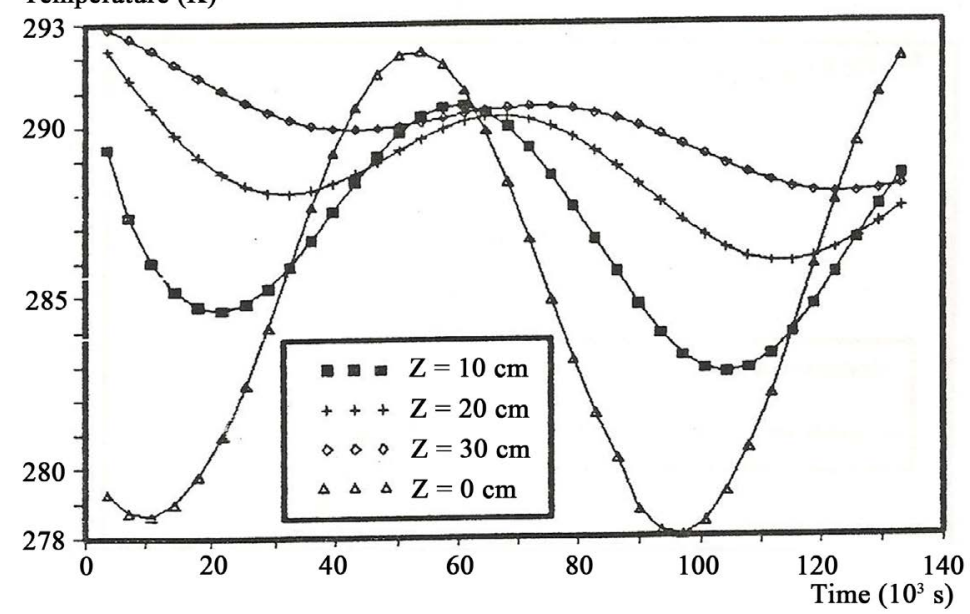

(b)

Figure 6. Temperature profiles with depth for a variable surface temperature, (a) On summer; (b) On winter. 
The heat flux on the soil surface is plotted in comparison with the fluxes at various depths in Figure 7 for one day in summer and winter. Their sinusoidal form as well as the presence of a dephasing is due mainly to the condition of the variable surface temperature and the damping of the propagating thermal wave. When the ambient temperature is higher than the ground temperature, the flux is positive and the medium absorbs heat, this often occurs during the day. During the night the flux becomes negative and the ground releases heat.

For a summer day, the heat flux is mainly positive and it reaches values as high as $0.025 \mathrm{~W} / \mathrm{cm}^{2}$ during the day, and it can release $0.015 \mathrm{~W} / \mathrm{cm}^{2}$ during the night. In contrast, in winter the temperature on the surface is lower than the initial temperature of ground, its effect is thus to cool the medium. Then for a winter day the heat flux is mainly negative, with values as low as $0.04 \mathrm{~W} / \mathrm{cm}^{2}$, during the day, and it absorbs $0.01 \mathrm{~W} / \mathrm{cm}^{2}$ during the night.

The comparison of the hydrous and concentration profiles obtained in the two types of boundary conditions of temperature in one day of summer (Figure 8) shows that they are almost identical. Indeed, the diffusion of heat is very slow; its influence is thus limited to the input of the column. At the moments when computations were made, $24 \mathrm{~h}$ and $36 \mathrm{~h}$, this part was almost saturated with water and aqueous solutions and the influence of the temperature is very small. Therefore the influence of a change in the boundary condition affects only the temperature.

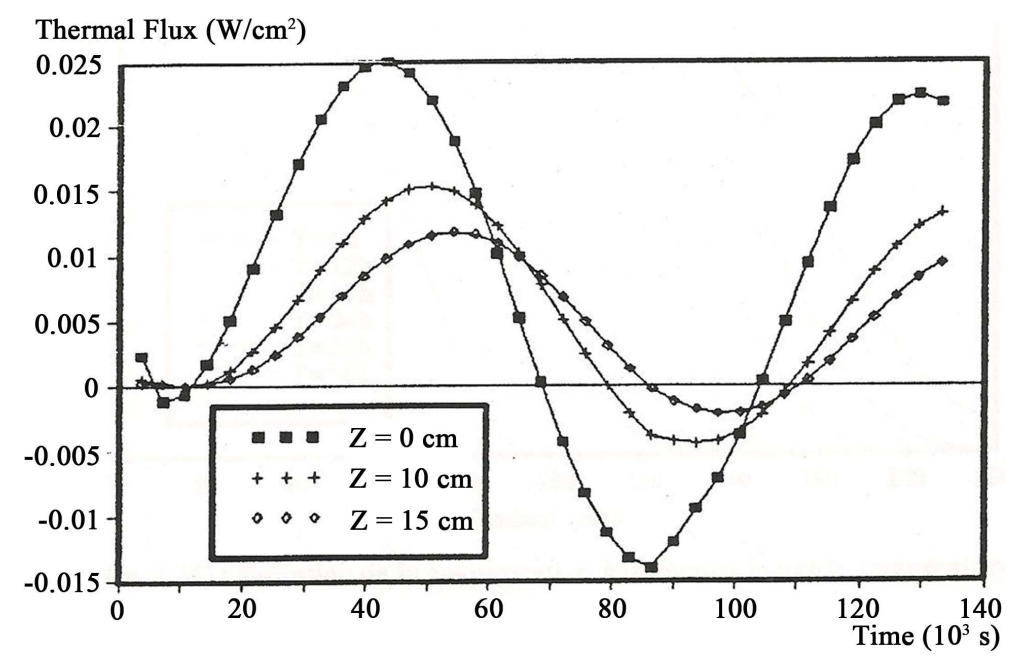

(a)

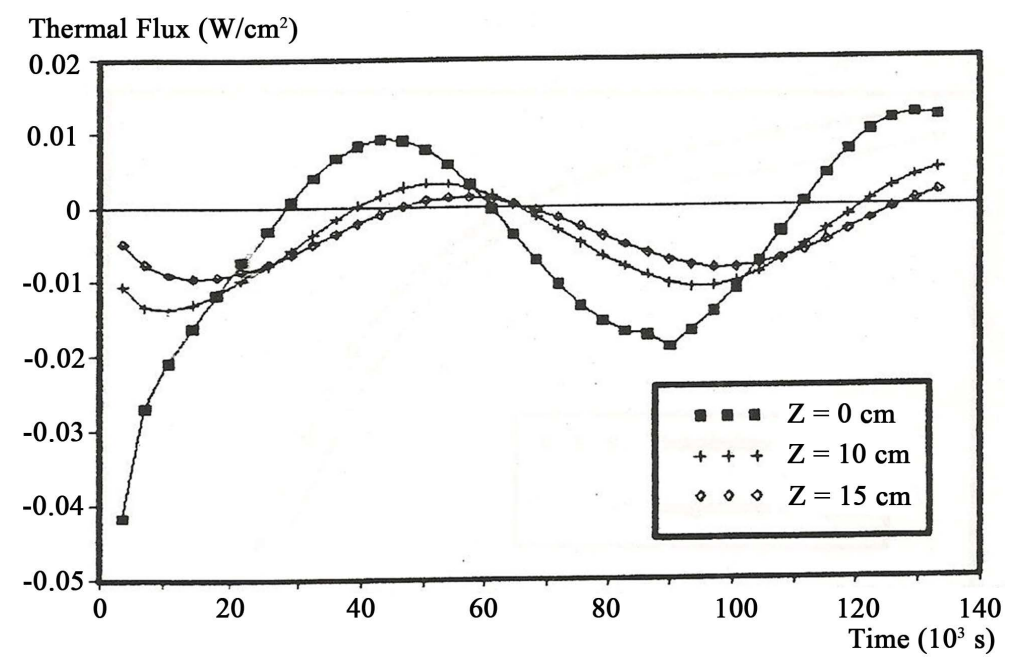

(b)

Figure 7. Heat flux profiles with depth for a variable surface temperature, (a) On summer; (b) On winter. 


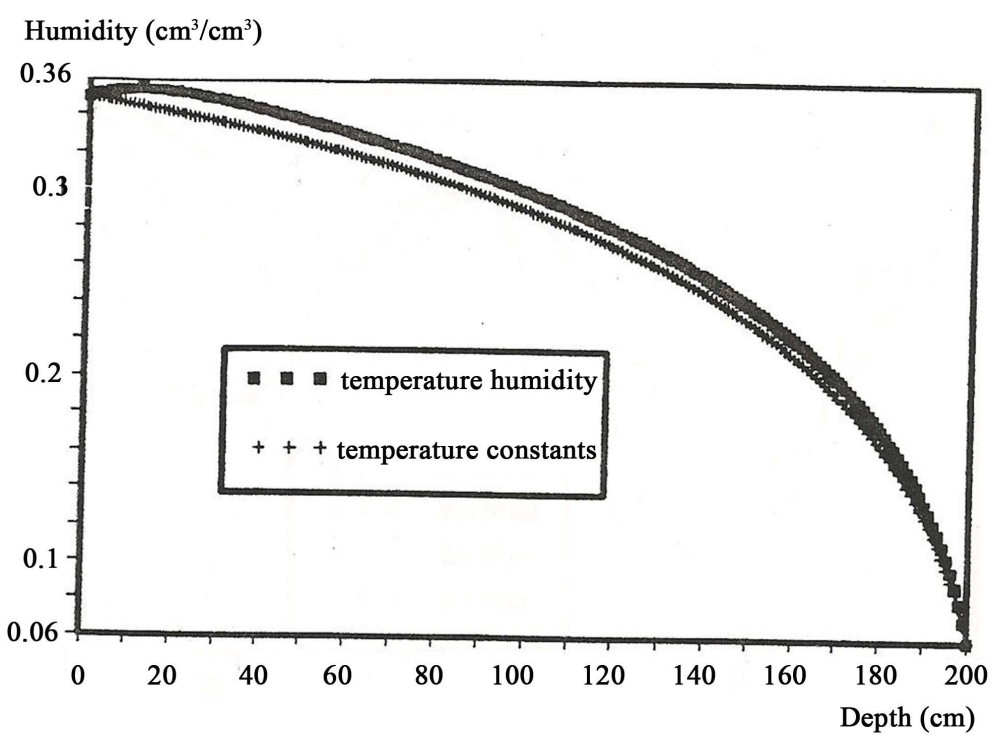

(a)

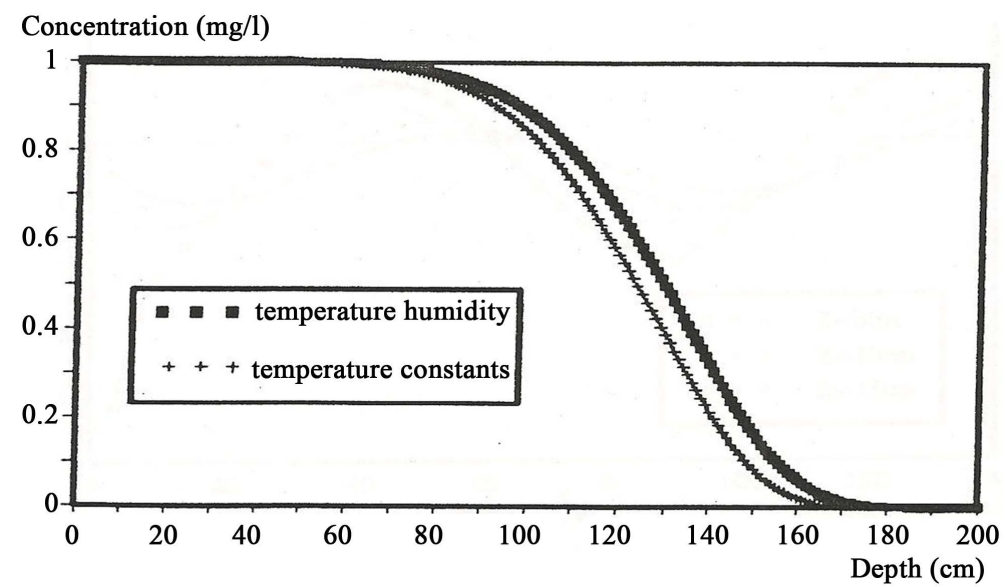

(b)

Figure 8. Comparison between curves at $\mathrm{t}=24 \mathrm{~h}$ for the two kinds boundary conditions (summer). (a) Humidity; (b) Contaminant concentration.

On the other hand, for one day of winter, the temperature gradient will be directed towards outside, in opposition with the hydrous and concentration gradients, being essential on their displacement. The transfer of water and pollutant in this case will be slower than in the case of constant temperature (Figure 9).

\section{Conclusions}

The numerical study of the coupled transfers of moisture, pollutant and heat was led in the case of a monodirectional flow. This study allowed us to follow the space-time evolution of the fronts of moistening, concentration and heat, and to study the influence of change of the boundary conditions on these fronts.

The results show that the heat transfer acts on the displacement of moisture, by migration of the hot zones towards the cold zones, which in its turn modifies the profiles of concentration.

During summer, the temperature is very high and close to the constant temperature. The effect of such a change is negligible and the profiles obtained are almost the same ones. However, in winter the temperature is low and the temperature gradient is directed towards outside. The change causes a deceleration of water and pollutant transport and the variation between the curves is important.

Therefore the effect of the sinusoidal condition of the temperature on the soil surface is more important during 


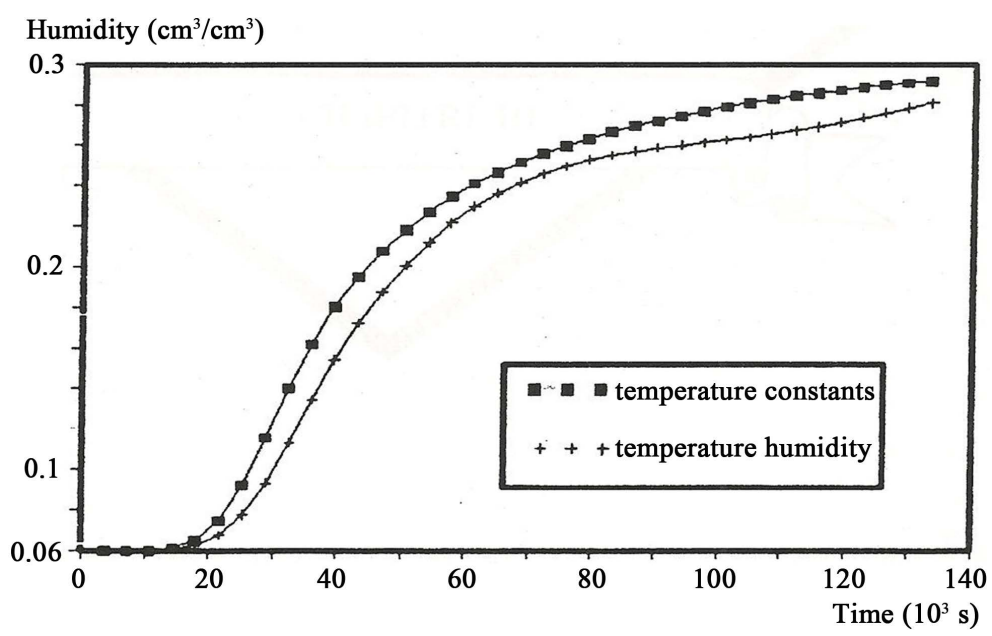

(a)

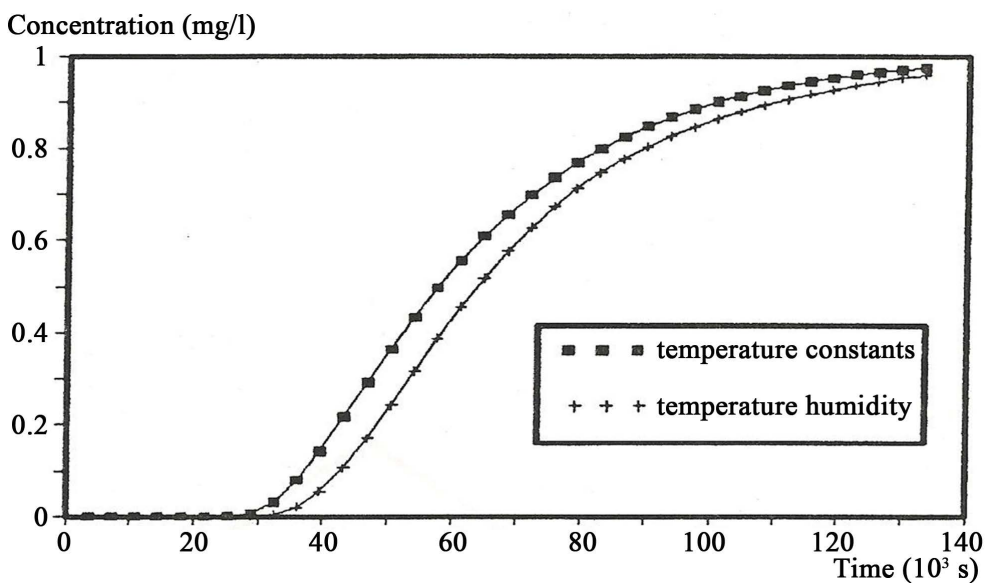

(b)

Figure 9. Comparison between temporal curves for the two kinds of boundary conditions (winter). (a) Humidity; (b) Contaminant concentration.

the winter than that during the summer, since the temperature gradient presents a great difference in both cases.

According to the results obtained, it seems that the adequate choice of the boundary conditions makes it possible to optimize the contributions of water and fertilizers in the presence of a temperature gradient.

\section{References}

[1] Gerson, H. and Mendes, N. (2006) Simultaneous Heat and Moisture Transfer in Soils Combined with Building Simulation. Energy and Buildings, 38, 303-314.

[2] Jury, W.A. (1982) Simulation of Solute Transport Using a Transfer Function Model. Water Resources Research, 18, 363-368.

[3] Liu, B.C., Liu, W. and Peng, S.W. (2005) Study of Heat and Moisture Transfer in Soil with a Dry Surface Layer. Intenational Journal of Heat and Mass Transfer, 48, 4579-4589.

[4] Viotti, P., Petrangeli Papini, M., Stracqualursi, N. and Gamba, C. (2005) Contaminant Transport in an Unsaturated Soil: Laboratory Test and Numerical Simulation Model as Procedure for Parameters Evaluation. Ecological Modeling, 183, 131-148. http://dx.doi.org/10.1016/j.ecolmodel.2004.07.014

[5] Jendele, L. (2002) An Improved Numerical Solution of Multiphase Flow Analysis in Soil. Advances in Engineering Software, 33, 659-668. http://dx.doi.org/10.1016/S0965-9978(02)00052-2

[6] El Hadji, B.D., et al. (2001) Modeling a Non-Conservative Solute Transfer in Unsaturated Porous Media. Earth and Planetary Sciences, 333, 129-132. 
[7] Van Genuchten, M.T. and Wierenga, P.J. (1997) Mass Transfer Studies in Sorbing Porous Media: II. Experimental Evaluation with Tritium. Soil Science Society of America Journal, 41, 272-278. http://dx.doi.org/10.2136/sssaj1977.03615995004100020022x

[8] Gaudet, J.P. (1978) Transfertd'eauet de solutésdans les sols non saturés, mesures et simulation. Ph.D. Thesis, University of Grenoble, Grenoble.

[9] Lewis, J. and Sjöstrom, J. (2010) Optimizing the Experimental Desing of Soil Columns in Saturated and Unsaturated Transport Experiments. Journal of Contaminant Hydrology, 115, 1-13. http://dx.doi.org/10.1016/j.jconhyd.2010.04.001

[10] Ibnoussina, M., El Haroui, M. and Maslouhi, A. (2006) Experimentation and Modeling of the Leaching of Nitric Nitrogen in a Sanly Soil. Comptes Rendus Geoscience, 338, 787-794. http://dx.doi.org/10.1016/j.crte.2006.07.002

[11] Patankar, S.V. (1978) Numerical Heat Transfer and Fluid Flow. McGraw-Hill, London.

[12] Philip, J. and De Vries, D.A. (1957) Moister Movement in Porous Materials under Temperature Gradients. Transactions, American Geophysical Union, 38, 2-22.

[13] Benyaich, M., Belghit, A., Gonzalez, A. and Claudet, B. (1998) Influence of Temperature on the Transport of Pollutant in Non-Saturated Soil. 3rd International Conference on Hydroscience and Engineering, Brandenburg University of Technology at Cottbus, ICHE, Cottbus/Berlin, 31 August-3 September 1998.

[14] Crausse, P. (1982) Etude fondamentale des transferts de chaleuretd'humidité. en milieu poreux non saturé. P.h.D., I.N.P. Toulouse.

[15] Ouardi, C. and Zrikem, Z. (1997) Sélection de séquencestypiques pour le climat de Marrakech: Application à l'habitat. 3ème Congrès de Mécanique, Tome II-b, Tetouan, 673-679.

[16] Pickens, J.F., Gilham, R.W. and Cameron, D.R. (1979) Finite Element Analysis of the Transport of Water and Solutes in the Drained Soils. Journal of Hydrology, 40, 243-264. http://dx.doi.org/10.1016/0022-1694(79)90033-7

\section{Nomenclature}

$\theta$ : Volumetric Water Contents

$q_{\theta}$ : Flux Density of Water

$K(\theta)$ : Hydraulic conductivity

$D_{\theta}$ : Isothermal Water Diffusivity

$D_{T}$ : Non-isothermal Water Diffusivity

Dap: Coefficient of Hydrodynamic Dispersion

$C$ : Concentration of pollutant

$\lambda$ : Thermal Conductivity

$C_{p}$ : Volumetric Heat Capacity of the Soil

$T$ : Temperature

z: Space Coordinate

$t$ : Time Coordinate 
Scientific Research Publishing (SCIRP) is one of the largest Open Access journal publishers. It is currently publishing more than 200 open access, online, peer-reviewed journals covering a wide range of academic disciplines. SCIRP serves the worldwide academic communities and contributes to the progress and application of science with its publication.

Other selected journals from SCIRP are listed as below. Submit your manuscript to us via either submit@scirp.org or Online Submission Portal.
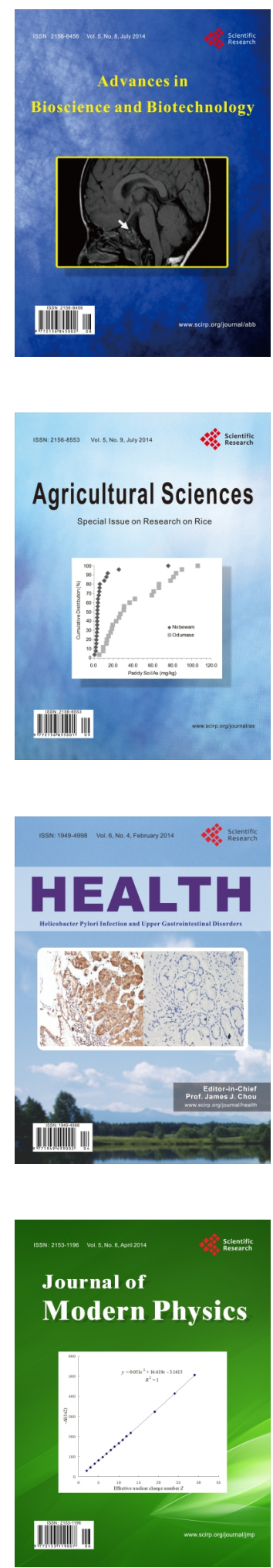
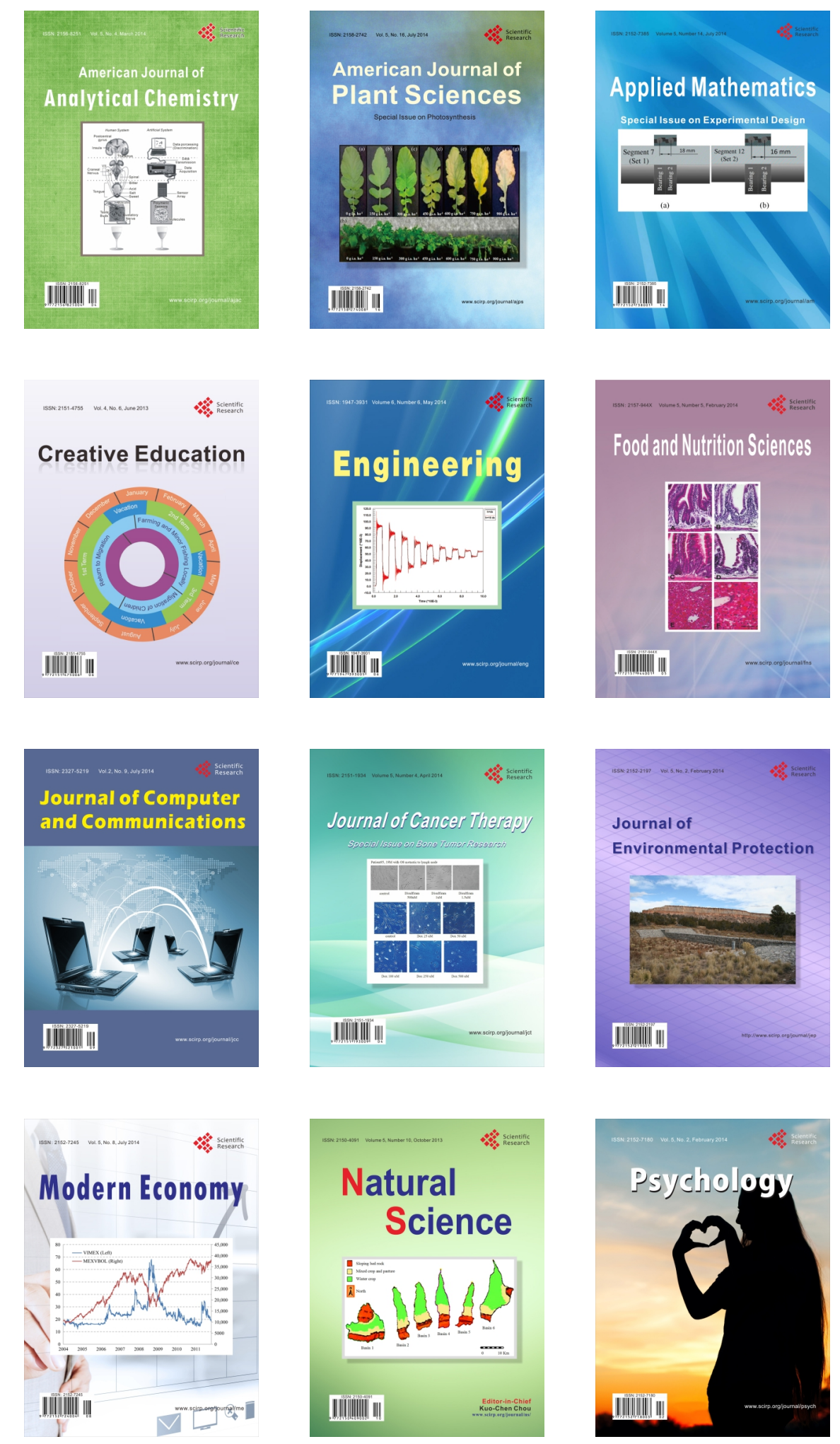